\title{
MULTIPLE KERNEL FUZZY CLUSTERING FOR UNCERTAIN DATA CLASSIFICATION
}

\author{
Nijaguna GS \\ Research Scholar, Visvesvaraya Technological University (VTU), Karnataka, India \\ Dr. Thippeswamy K \\ Professor \& Head, Department of Computer Science Engineering, \\ Visvesvaraya Technological University, Regional Center Mysuru, India
}

\begin{abstract}
:
Traditionallcall tree classifiers worklwith information whose valueslarea unit celebrated and precise. Welhave a tendency to extendlsuch classifiers to handle information1with unsure info. Worth uncertaintylarises in several applications throughoutthelinformationassortmentmethod.Examplelsource of uncertainty embracelmeasurement/quantization errors, information staleness, and multiple recurrent measurements. Withluncertainty, the worth of a knowledge item lis commonly depicted not by one1single worth, however bylmultiple values forming a probability distribution. Instead oflabstracting unsure information by appliedlmath derivatives (such aslmean and median), we have altendency to discover thatlthe accuracy of a call1tree classifier will be a lotlof improved if the "completelinformation" of a knowledge item is utilized. Sincelprocess pdf's is computationall1 a lot of pricey thanlprocess single values (e.g., averages), call treelconstruction on unsure informationl is more electronic equipmentldemanding than that sure information. Toltackle this problem, we have a tendencyl to propose a series of pruningltechniques which will greatly improvelconstruction potency.
\end{abstract}

Key words: Embrace, Probability distribution.

Cite this Article: Nijaguna GS and Dr. Thippeswamy K, Multiple Kernel Fuzzy Clustering for Uncertain Data Classification, International Journal of Computer Engineering and Technology, 10(01), 2019, pp. (253)-(261). http://www.iaeme.com/IJCET/issues.asp?JTypeIJCET\&VType=10\&IType=1

\section{INTRODUCTION}

A. Data Mining: Data mining is the extraction of hidden predictive information from large databases, is a powerful new technology with great potential to help companies focus on the most important information in their data warehouses. Data mining tools predict future trends and behaviors, allowing businesses to make proactive, knowledge-driven decisions. The automated, prospective analyses offered by data mining move beyond the analyses of 
past events provided by retrospective tools typical of decision support systems. Data mining tools can answer business questions that traditionally were too time consuming to resolve. They scour databases for hidden patterns, finding predictive information that experts may miss because it lies outside their expectations. Most companies already collect and refine massive quantities of data. . The process of applying a model to new data is known as scoring. Many forms of data mining are predictive. For example, a model might predict income based on education and other demographic factors. Predictions have an associated probability. Prediction probabilities are also known as confidence. Other forms of data mining identify natural groupings in the data. For example, a model might identify the segment of the population that has an income within a specified range, that has a good driving record, and that leases a new car on a yearly basis. Data mining can derive actionable information from large volumes of data. For example, a town planner might use a model that predicts income based on demographics to develop a plan for low-income housing.

B. The Scope of Data Mining: Datalmining derives its name from the similarities between searching for valuable business information in a large database - for example, finding linked products in gigabytes of store scanner data - and mining a mountain for a vein of valuable ore. Both processes require either sifting through an immense amount of material, or Intelligently probing it to find exactly where the value resides. Given databases of sufficient size and quality, data mining technology can generate new business opportunities by providing these capabilities:

$>$ Automated prediction of trends and behaviors. Data mining automates the process of finding predictive information in large databases. Questions that traditionally required extensive hands-on analysis can now be answered directly from the data quickly. A typical example of a predictive problem is targeted marketing. Data mining uses data on past promotional mailings to identify the targets most likely to maximize return on investment in future mailings. Other predictive problems include forecasting bankruptcy and other forms of default, and identifying segments of a population likely to respond similarly to given events.

$>$ Automated discovery of previously unknown patterns. Data mining tools sweep through databases and identify previously hidden patterns in one step. An example of pattern discovery is the analysis of retail sales data to identify seemingly unrelated products that are often purchased together. Other pattern discovery problems include detecting fraudulent credit card transactions and identifying anomalous data that could represent data entry keying errors.

\section{DECISION TREE}

Decision tree learning is a method commonly used in data mining. The goal is to create a model that predicts the value of a target variable based on several input variables. An example is shown on the right. Each interior node corresponds to one of the input variables; there are edges to children for each of the possible values of that input variable. Each leaf represents a value of the target variable given the values of the input variables represented by the path from the root to the leaf. A tree can be "learned" by splitting the source set into subsets based on an attribute value test. This process is repeated on each derived subset in a recursive manner called recursive partitioning. The recursion is completed when the subset at a node has all the same value of the target variable, or when splitting no longer adds value to the predictions. This process of top-down induction of decision trees (TDIDT) is an example of a greedy algorithm, and it is by far the most common strategy for learning decision trees from data, but it is not the only strategy. In fact, some approaches have been 
developed recently allowing tree induction to be performed in a bottom-up fashion. In data mining, decision trees can be described also as the combination of mathematical and computational techniques to aid the description, categorization and generalization of a given set of data.

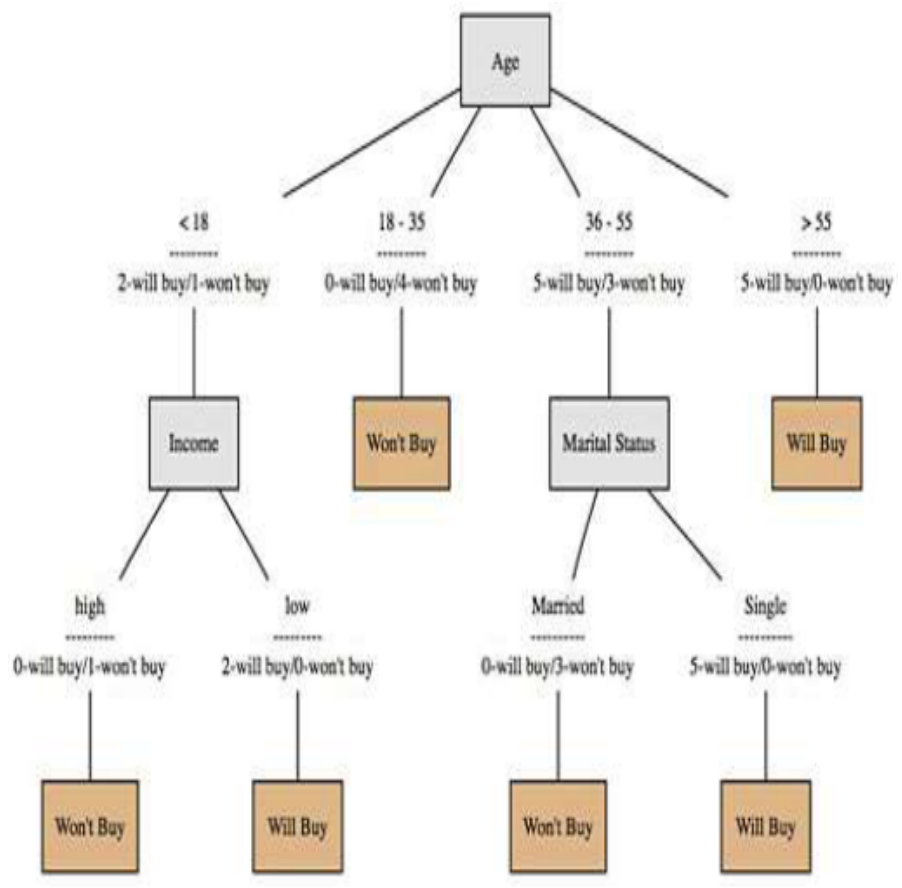

Figure 1: Sample Decision Tree

\section{ALGORITHM}

\subsection{C4.5 ALGORITHM}

C4.5 is a calculation used to produce a decision tree created by Ross Quinlan.[1] C4.5 is an augmentation of Quinlan's prior ID3 calculation. The decision trees created by C4.5 can be utilized for characterization, and therefore, $\mathrm{C} 4.5$ is regularly alluded to as a factual classifier. C4.5 constructs decision trees from an arrangement of preparing information similarly as ID3, utilizing the idea of data entropy. The preparation information is an arrangement of effectively ordered examples. Each sample comprises of a p-dimensional vector , where they speak to traits or highlights of the example, and also the class in which falls.

At every hub of the tree, C4.5 picks the property of the information that most successfully parts its arrangement of tests into subsets enhanced in one class or the other. The part rule is the standardized data pick up (contrast in entropy). The characteristic with the most noteworthy standardized data pick up is settled on the decision. The C4.5 calculation then recurses on the littler sublists.

This calculation has a couple of base cases.

D All the samples in the list belong to the same class. At the point when this happens, it basically makes a leaf hub for the decision tree saying to pick that class.

$>$ None of the features give any data pick up. For this situation, C4.5 makes a decision hub higher up the tree utilizing the normal estimation of the class.

Instance of previously unseen class encountered. Once more, C4.5 makes a decision hub higher up the tree utilizing the expected value. 


\subsection{ENTROPY}

Entropy is a measure of unpredictability or information content. To get an informal, intuitive understanding of the connection between these three English terms, consider the example of a poll on some political issue. Usually, such polls happen because the outcome of the poll isn't already known. In other words, the outcome of the poll is relatively unpredictable, and actually performing the poll and learning the results gives some new information; these are just different ways of saying that the entropy of the poll results is large. Now consider the case that the same poll is performed a second time shortly after the first poll. Since the result of the first poll is already known, the outcome of the second poll can be predicted well and the results should not contain any new information; in this case the entropy of the second poll results is small. Now consider the example of a coin toss. When the coin is fair, that is, when the probability of heads is the same as the probability of tails, then the entropy of the coin toss is as high as it could be. This is because there is no way to predict the outcome of the coin toss ahead of time - the best we can do is predict that the coin will come up heads, and our prediction will be correct with probability $1 / 2$. Such a coin toss has one bit of entropy since there are two possible outcomes that occur with equal probability, and learning the actual outcome contains one bit of information. Contrarily, a coin toss with a coin that has two heads and no tails has zero entropy since the coin will always come up heads, and the outcome can be predicted perfectly. Most collections of data in the real world lie somewhere in between.

English text has fairly low entropy. In other words, it is fairly predictable. Even if we don't know exactly what is going to come next, we can be fairly certain that, for example, there will be many more e's than z's, or that the combination 'qu' will be much more common than any other combination with a ' $q$ ' in it and the combination 'th' will be more common than ' $z$ ', ' 'q', or 'qu'. Uncompressed, English text has about one bit of entropy for each character (commonly encoded as eight bits) of message.

If a compression scheme is lossless - that is, you can always recover the entire original message by decompressing - then a compressed message has the same quantity of information as the original, but communicated in fewer bits. That is, it has more information per bit, or a higher entropy. This means a compressed message is more unpredictable, because there is no redundancy; each bit of the message is communicating a unique bit of information. Roughly speaking, Shannon's source coding theorem says that a lossless compression scheme cannot compress messages, on average, to have more than one bit of information per bit of message. The entropy of a message multiplied by the length of that message is a measure of how much information the message contains.

\subsection{GAIN RATIO}

Data delivered by information mining strategies can be represented in a wide range of ways. Decision tree structures are a typical approach to sort out arrangement plans. In ordering assignments, decision trees visualize what steps are taken to arrive at a characterization. Each decision tree starts with what is named a root hub, thought to be the "parent" of each other hub. Every hub in the tree assesses a characteristic in the information and figures out which way it ought to take after. Normally, the decision test depends on contrasting an value against some constant. Characterization utilizing a decision tree is performed by directing from the root hub until arriving at a leaf hub.

The delineation gave here is a cannonical case in information mining, including the decision to play or not play in light of atmosphere conditions. For this situation, standpoint is in the position of the root hub. The degrees of the hub are quality esteems. In this case, the child nodes are trial of mugginess and blustery, prompting the leaf hubs 
which are the real classification. This illustration additionally incorporates the relating information, likewise alluded to as occasions. In our case, there are 9 "play" days and 5 "no play" days.

Decision trees can represent different sorts of information. The least difficult and most well-known is numerical information. It is regularly attractive to compose regular information also. Nominal quantities are formally portrayed by a discrete arrangement of images. For example, climate can be portrayed in either numeric or nominal mold. We can measure the temperature by saying that it is 11 degrees Celsius or 52 degrees Fahrenheit. We could likewise say that it is icy, cool, mellow, warm or hot. The previous is a case of numeric information, and the last is a sort of nominal information. More precisely, the case of chilly, cool, gentle, warm and hot is an extraordinary sort of nominal information, portrayed as ordinal information. Ordinal information has an understood supposition of requested connections between the qualities. Proceeding with the climate case, we could likewise have a simply nominal depiction like bright, cloudy and stormy. These qualities have no connections or separation measures.

The kind of information sorted out by a tree is essential for understanding how the tree functions at the hub level. Reviewing that every hub is successfully a test, numeric information is regularly assessed in terms of simple mathematical inequality. For example, numeric climate information could be tried by finding on the off chance that it is more prominent than 10 degrees Fahrenheit. Nominal information is tried in Boolean design; as it were, regardless of whether it has a specific value. The representation demonstrates the two sorts of tests. In the climate illustration, viewpoint is an nominal information type. The test essentially asks which quality value is represented and routes accordingly. The mugginess hub reflects numeric tests, with a disparity of less than or equivalent to 70, or more prominent than 70 .

Decision tree enlistment calculations work recursively. Initial, a attribute must be chosen as the root hub. With a specific end goal to make the most proficient (i.e, littlest) tree, the root hub should successfully part the information. Each split attempts to pare down an arrangement of occurrences (the genuine information) until the point that they all have a similar grouping. The best split is the one that gives what is named the most data gain.

Data in this setting originates from the idea of entropy from information theory, as created by Claude Shannon. Despite the fact that "data" has numerous unique situations, it has a very specific mathematical meaning relating to certainty in decision making. In a perfect world, each split in the decision tree ought to convey us more like a characterization. One approach to conceptualize this is to see each progression along the tree as removing randomness or entropy. Data, communicated as a numerical amount, reflests this. For instance, consider an extremely basic grouping issue that requires making a decision tree to choose yes or no based on few information. This is precisely the situation visualized in the decision tree. Every characteristic values will have a specific number of yes or no orders. On the off chance that there are equivalent quantities of yeses and no's, at that point there is a lot of entropy in that value. In this circumstance, data achieves a greatest. Alternately, if there are just yeses or just no's the data is additionally zero. The entropy is low, and the test value is exceptionally valuable for settling on a decision. The formula for calculating intermediate alues is as follows:

$$
\text { Info }=-\sum_{i=1}^{m} p_{1} \log _{2} p_{1} \ldots
$$

How about we separate this. Consider attempting to compute the data pick up for three factors for one attribute. The attribute overall has an aggregate of nine yeses and five no's. 
The main variable has two yeses and three no's. The second has four yeses and zero no's. The last has three yeses and two no's. Our initial step is to calculate the data for each of the factors.

Starting with the first, our formula leads us to info([2,3]) being equal to $-2 / 5 \times \log 2 / 5$ - $3 / 5 \times \log 3 / 5$. This comes to 0.971 bits. Our second variable is easy to calculate. It only has yeses, so it has a value of 0 bits. The final variable is just the reverse of the first -- the value is also 0.971 bits. Having found the information for the variables, we need to calculate the information for the attribute as a whole: 9 yeses and 5 no's. The calculation is info $([9,5])=-9 / 14 \times \log 9 / 14-5 / 14 \times \log 5 / 14$. This comes to 0.940 bits.

In decision tree induction, our objective is to find the overall information gain. This is found by averaging the information value of the attribute values.In our case, this is equivalent to finding the information of all the attributes together.

We would use the formula

info $([2,3],[4,0],[3,2])=(5 / 14) \times 0.971+(4 / 14) \times 0+(5 / 14) \times 0.971$. This comes to 0.6931 bits.

The final step is to calculate the overall information gain. Information gain is found by subtracting the information value average by the raw total information of the attribute. Mathematically, we would calculate gain $=\operatorname{info}([9,5])-\operatorname{info}([2,3],[4,0],[3,2])=0.940-0.693$ $=0.247$. The decision tree acceptance calculation will figure this total for each property, and select the one with the most elevated data pick up as the root hub, and proceed with the estimation recursively until the point when the information is totally grouped. This approach is one of the principal strategies utilized for decision tree enlistment. It has various conceivable weaknesses. One normal issue emerges when a characteristic has an extensive number of extraordinarily recognizing values. A case of this could be standardized savings numbers, or different sorts of individual recognizable proof numbers. For this situation, there is a misleadingly high decision value to the data - the ID classifies every single individual, and distorts the calculation by over fitting the information. One arrangement is to utilize a data pick up proportion that predispositions attributes with vast quantities of particular values.

\subsection{PRUNING}

One of the questions that arises in a decision tree algorithm is the optimal size of the final tree. A tree that is too large risks overfitting the training data and poorly generalizing to new samples. A small tree might not capture important structural information about the sample space. However, it is firm to tell when a tree algorithm should stop because it is impossible to tell if the addition of a single extra node will dramatically decrease error. This problem is known as the horizon effect. A common strategy is to grow the tree until each node contains a small number of instances then use pruning to remove nodes that do not provide additional information. [1] Pruning should reduce the size of a learning tree without reducing predictive accuracy as measured by a test set or using cross-validation. There are many techniques for tree pruning that differ in the measurement that is used to optimize performance. 


\section{SYSTEM ARCHITECTURE}

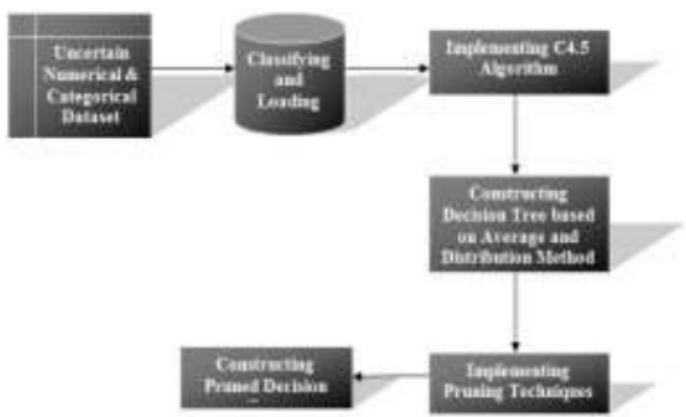

Figure 2 System Architecture

\subsection{Modules Specification}

Modules:

$>$ Processing Dataset.

$>$ Detecting Split Points

$>$ Decision tree construction.

$>$ Pruning algorithm implementation.

$>$ Performance analysis.

Processing Dataset:

$>$ Selecting the numerical dataset from UCI repository.

$>$ Loading the selected dataset into database.

$>$ Classifying dataset based on class attributes in the dataset.

$>$ Loading classified dataset in the database for calculation.

Detecting Split Points:

$>$ Calculating entropy for all attributes.

$>$ Calculating Info and gain for all the attributes in the dataset.

$>$ Selecting the best split points with maximum gain.

$>$ Sorting the values based on gain.

Decision Tree Construction:

Sorting the attributes with less probability.

$>$ Classifying the attributes based on its probability values.

$>$ Sorting the attributes with highest mean value.

$>$ Classifying the attributes based on its mean values.

$>$ Constructing the decision tree with selected attributes.

Pruning Algorithm Implementation:

$>$ Finding set of end-points for tuples in attribute $\mathrm{Aj}$.

$>$ Computing Entropy for all end-points for all $\mathrm{k}$ attributes.

$>$ Finding the pruning threshold.

$>$ Finding best split points on attribute $\mathrm{Aj}$.

$>$ Pruning the end-points for all $\mathrm{k}$ attributes. 
Performance Analysis:

Calculating the execution time for various pruning algorithm.

$>$ Finding the number of entropy calculation by implementing various pruning algorithm.

Evaluating the effectiveness of various pruning algorithm.

$>$ Evaluating the effects of samples and width for attribute.

$>$ Performance analysis by Comparing the results of various pruning algorithm

\subsection{Flow Chart}

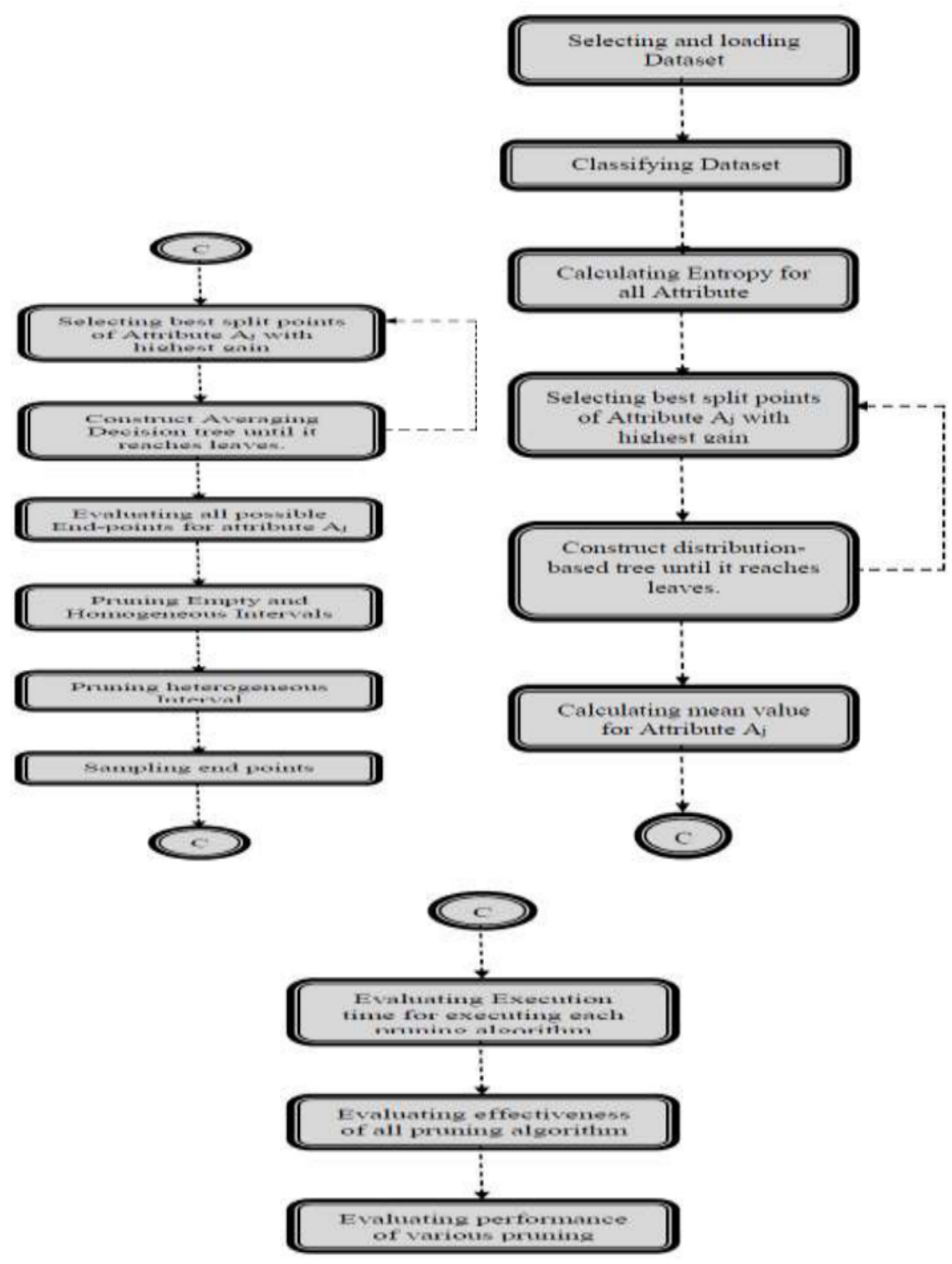




\section{CONCLUSION}

We have extended the model of decision-tree classification to accommodate knowledge tuples having numerical attributes with insecurity delineated by discretional pdf's. We've changed classical call tree building algorithms (based on the structure of C4.5[3]) to create call trees for classifying such data. We've found by trial and error that once appropriate pdf's area unit used, exploiting knowledge uncertainty ends up in call trees with remarkably higher accuracies. We tend to thus advocate that knowledge be collected and hold on with the pdf info intact. Performance is a problem, though, owing to the raised quantity of information to be processed, similarly because the additional difficult entropy computations concerned. Therefore, we've devised a series of pruning techniques to enhance tree construction efficiency. Our algorithms are by experimentation verified to be extremely effective. Their execution times area unit of associate order of magnitude such as classical algorithms. Some of these pruning technique area unit generalizations of analogous techniques for handling point valued knowledge. Different techniques, namely pruning by bounding and end-point sampling area unit novel. Though our novel techniques area unit primarily designed to handle unsure knowledge, they're additionally helpful for building call trees exploitation classical algorithms once there area unit tremendous amounts of knowledge tuples.

\section{REFERENCES}

[1] M. Chau, R. Cheng, B. Kao, and J. Ng, "Uncertain data mining: An example in clustering location data," in PAKDD, ser. Lecture Notes in Computer Science, vol. 39 8. Singapore: Springer, 9-12 Apr. 2006, pp. 199-204.

[2] S. D. Lee, B. Kao, and R. Cheng, "Reducing UK-means to K-means," The 1st Workshop on Data Mining of Uncertain Data (DUNE), in conjunction with the $7^{\text {th }}$ IEEE International Conference on Data Mining (ICDM

[3] K. Chui, B. Kao, and E. Hung, "Mining frequent itemsets from uncertain data," PAKDD, ser. Lecture Notes in Computer Science, vol. 4426. Nanjing, China: Springer, 22-25 May 2007, pp. 47-58.), Omaha, NE, USA, 28 Oct. 2007.

[4] L. Breiman, "Technical note: Some properties of splitting criteria", Machine Learning, vol. 24, no. 1, pp. 41-47, 1996.

[5] A. Nierman and H. V. Jagadish, "ProTDB: Probabilistic data in XML", in VLDB. Hong Kong, China: Morgan Kaufmann, 20-23 Aug. 2002, pp. 646-657.

[6] J. Chen and R. Cheng, "Efficient evaluation of imprecise location dependent queries," in ICDE. Istanbul, Turkey: IEEE, 15-20 Apr. 2007, pp. 586-595.

[7] M. Chau, R. Cheng, B. Kao, and J. Ng, "Uncertain data mining: An example in clustering location data," in PAKDD, ser. Lecture Notes in Computer Science, vol. 3918. Singapore: Springer, 9-12 Apr. 2006, pp. 199-204.

[8] R. Cheng, Y. Xia, S. Prabhakar, R. Shah, and J. S. Vitter, "Efficient indexing methods for probabilistic threshold queries over uncertain data," in VLDB. Toronto, Canada: Morgan Kaufmann, Transactions on Knowledge and Data Engineering, 31 Aug.-3 Sept.2004, pp. $876-887$.

[9] R. Cheng, D. V. Kalashnikov, and S. Prabhakar, "Querying imprecisedata in moving object environments," IEEE Trans. Knowl. Data Engineering, vol. 16, no. 9, pp. 1112-1127, 2004.

[10] W. K. Ngai, B. Kao, C. K. Chui, R. Cheng, M. Chau, and K. Y. Yip, "Efficient clustering of uncertain data," ICDM. Hong Kong, China: IEEE Computer Society, 18-22 Dec. 2006, pp. 436-445.

[11] S. D. Lee, B. Kao, and R. Cheng, "Reducing UK-means to K-means," in the $1^{\text {st }}$ Workshop on Data Mining of Uncertain Data (DUNE), inconjunction with the 7th IEEE International Conference on Data Mining (ICDM), Omaha, NE, USA, 28 Oct. 2007. 